\title{
A NEW PROOF OF THE STRONG PARTITION RELATION ON $\omega_{1}$
}

\author{
STEVE JACKSON
}

\begin{abstract}
Assuming the axiom of determinacy, we give a new proof of the strong partition relation on $\omega_{1}$. The proof is direct and avoids appeal to complicated set-theoretic machinery.
\end{abstract}

\section{INTRODUCTION}

We give a new proof of the strong partition relation (defined below) on $\omega_{1}$, denoted by $\omega_{1} \rightarrow\left(\omega_{1}\right)^{\omega_{1}}$, assuming the axiom of determinacy. This result is, in itself, not new but is a theorem of Martin (see [Martin 1]). In fact, a second, quite different, proof of this partition relation was obtained later by Kechris (see [Kechris 1]). However, both of these proofs used techniques relying heavily on the special nature of $\omega_{1}$. Martin's proof relies on the theory of indiscernibles for the models $L[x]$, while Kechris's proof appeals to the notion of generic codes for countable ordinals. More recently, Kechris and Woodin (see [Kechris-Woodin]) have extended the theory of generic codes to uncountable ordinals allowing them to obtain weaker partition relations for the higher $\delta_{n}^{1}$ 's (see [Moschovakis] for the definition of the $\delta_{n}^{1}$ 's and development of descriptive set theory). However, neither of these methods seems able to yield the full strong partition relation on the higher odd projective ordinals, $\boldsymbol{\delta}_{2 n+1}^{1} \rightarrow\left(\boldsymbol{\delta}_{2 n+1}^{1}\right)^{\boldsymbol{\delta}_{2 n+1}^{1}}$. One feature of our proof is that the methods used here, when appropriately generalized and combined with a more detailed analysis of the projective ordinals, suffice to get the strong partition relation on all the odd projective ordinals. The main part of the detailed analysis of the projective hierarchy has been written up, and appears in [Jackson 2]. However, a second part, in which the analysis is used to establish the partition relations (which are necessary in the complete inductive analysis), and certain other things, remains to be written up. Our proof here allows one to see in a simple setting some of the extra ideas involved, without getting involved with excessive technical complexity. Also, our proof is quite "elementary" and self-contained, and does not appeal to more complicated set-theoretic machinery.

Received by the editors April 18, 1988 and, in revised form, October 27, 1988.

1980 Mathematics Subject Classification (1985 Revision). Primary 03E60, $03 E 15$.

Key words and phrases. Determinacy, strong partition relation.

The author was partially supported by an NSF fellowship. 
Historically, the analysis of [Jackson 1] (where the initial goal was to calculate the ordinal $\delta_{5}^{1}$ ) preceded the current proof. Martin then noticed that the methods developed there sufficed to give a good enough coding of the subsets of $\delta_{3}^{1}$ to yield a proof of the strong partition relation on $\delta_{3}^{1}$. When isolated, these methods in turn yielded a new, simple proof of the strong partition relation on $\omega_{1}$.

There are two basic ideas used in our proof. The first is to analyze directly the measures on $\omega_{1}$, where by measure we mean a countably additive ultrafilter. It is this step, relatively simple here, which becomes technically much more difficult when considering $\boldsymbol{\delta}_{3}^{1}$. The second idea, due to Kunen, enables one to convert an analysis of the measures on $\omega_{1}$ into a good coding of the subsets of $\omega_{1}$ by reals. Kunen used this idea to show (from AD again) the weak partition relation on $\boldsymbol{\delta}_{3}^{1}$, i.e. $\boldsymbol{\delta}_{3}^{1} \rightarrow\left(\boldsymbol{\delta}_{3}^{1}\right)^{\lambda}$ for all $\lambda<\boldsymbol{\delta}_{3}^{1}$ (see [Solovay]).

Throughout this paper, we work in the theory $\mathrm{ZF}+\mathrm{AD}+\mathrm{DC}$. We recall some basic definitions. We let $\omega^{\omega}$ denote the set of "reals", i.e. infinite sequences of natural numbers $x=(x(0), x(1), \ldots, x(n), \ldots)$. To each $A \subseteq \omega^{\omega}$ we associate a two player game, $G_{A}$, in which players I and II alternately pick natural numbers, eventually producing a real $x$ :

$$
\text { I } x(0) \quad x(1){ }^{x(2)} x(3) \quad x=(x(0), x(1), \ldots)
$$

Then I wins the game iff $x \in A$.

The axiom of determinacy, $\mathrm{AD}$, asserts that for every $A$, the game $G_{A}$ is determined, that is, one of the players has a winning strategy, with the usual meaning. DC, the axiom of dependent choices, asserts that every ill-founded binary relation has an infinite descending chain.

By the strong partition relation on $\omega_{1}, \omega_{1} \rightarrow\left(\omega_{1}\right)^{\omega_{1}}$, we mean the assertion that for any partition $F:\left[\omega_{1}\right]^{\omega_{1}} \rightarrow\{0,1\}$ (where here $[X]^{\omega_{1}} \equiv$ the set of all increasing functions from $\omega_{1}$ to $\left.X\right)$ there is an uncountable $A \subseteq \omega_{1}$ and $i \in\{0,1\}$ with $F(f)=i$ for all $f \in[A]^{\omega_{1}}$. This is easily seen to be equivalent to another version, somewhat easier to work with, which we now state. We say a function $f: \omega_{1} \rightarrow \omega_{1}$ is of the correct type if there is an increasing $\tilde{f}: \omega_{1} \rightarrow \omega_{1}$ such that $f(\alpha)=\sup _{\beta<\omega \cdot(\alpha+1)} \tilde{f}(\beta)$. (Intuitively, this says that $f$ is increasing, noncontinuous, and uniformly has range in points of cofinality $\omega$.$) Then, \omega_{1} \rightarrow\left(\omega_{1}\right)^{\omega_{1}}$ if for all partitions $F:\left\{f: \omega_{1} \rightarrow \omega_{1}\right.$ of the correct type $\} \rightarrow\{0,1\}$, there is a c.u.b. $C \subseteq \omega_{1}$ and an $i \in\{0,1\}$ such that $F(f)=i$ for all $f: \omega_{1} \rightarrow C$ of the correct type.

We may similarly define the partition relations $\omega_{1} \rightarrow\left(\omega_{1}\right)^{\lambda}$ for any $\lambda<\omega_{1}$ using functions $f: \lambda \rightarrow \omega_{1}$ of the correct type-with obvious meaning.

We will use throughout the fact that $\omega_{1} \rightarrow\left(\omega_{1}\right)^{n}$ for all $n \in \omega$, where here we mean the above (second) form of the partition relation. We refer the reader to [Kechris 2] for proofs. In particular, it follows that $\omega_{1}$ is measurable and the c.u.b. filter is a normal measure on $\omega_{1}$-in fact the unique normal measure on $\omega$. 


\section{ANALYSIS OF THE MEASURES ON $\omega_{1}$}

We get a normal form for the measures on $\omega_{1}$. We begin by considering an arbitrary measure $\nu=\nu_{0}$ on $\omega_{1}$. Recall that (with AD) all measures are countably additive. We assume $\nu$ is nonprincipal for the moment. Let $f_{0}: \omega_{1} \rightarrow \omega_{1}$ be a representative of the least equivalence class with respect to $\nu$ of a function which is almost everywhere (not strictly) pressing down, nonconstant and monotonically increasing. That is, $f_{0}$ is not constant on any measure one set (with respect to $\nu$ ), and there is a measure one set $A \subseteq \omega_{1}$ such that $f(\alpha) \leq \alpha$ for all $\alpha \in A$ and $\alpha<\beta, \alpha, \beta \in A$ implies $f(\alpha) \leq f(\beta)$. We define the measure $\bar{\nu}$ by $\bar{\nu}(A)=1$ iff $\nu\left(\left(f_{0}^{-1}\right)^{\prime \prime} A\right)=1$, in other words, $\bar{\nu}(A)=1$ iff for almost all $\alpha$ with respect to $\nu, f_{0}(\alpha) \in A$. We abbreviate this by writing $\bar{\nu}=f_{0}(\nu)$.

Claim. $\bar{\nu}$ is the normal measure on $\omega_{1}$.

Proof. If not, we let $C \subseteq \omega_{1}$ be c.u.b. and $A \subseteq \omega_{1}$ have measure one with respect to $\bar{\nu}$ be such that $A \cap C=\varnothing$. We define $g: A \rightarrow \omega_{1}$ by $g(\alpha)=$ the largest element of $C<\alpha$. However, $g \circ f_{0}: \omega_{1} \rightarrow \omega_{1}$ now violates the minimality of $f_{0}$.

For notational convenience, we let $N$ denote the normal measure on $\omega_{1}$ given by the c.u.b. filter. We fix $A_{0} \subseteq \omega_{1}$ to be a measure one set with respect to $\nu$ on which $f_{0}$ is monotonically increasing and (nonstrictly) pressing down. Let $g_{0}: \omega_{1} \rightarrow \omega_{1}$ be defined by $g_{0}(\alpha)=\sup \{\beta \in A: f(\beta) \leq \alpha\}$. Then $g$ is monotonically increasing and $g_{0}$ dominates $\nu$ with respect to $N$, i.e., there is a c.u.b. $C \subseteq \omega_{1}$ (so $\left.N(C)=1\right)$ such that $\left\{\alpha<\omega_{1}\right.$ : for some $\beta \in C$, $\beta \leq \alpha \leq g(\beta)\}$ has measure one with respect to $\nu$. We fix such a c.u.b. $C_{0} \subseteq \omega_{1}$.

We digress now for a moment to recall some facts, due to Kunen, which permit an analysis of functions from $\omega_{1}$ to $\omega_{1}$. Let $\mathrm{WO} \subseteq \omega^{\omega}$ be the canonical set of reals coding well-orderings of $\omega$. So, WO is $\Pi_{1}^{1}$-complete. Let $T_{1} \subseteq\left(\omega \times \omega_{1}\right)^{<\omega}$ be the tree defined by $(s, \vec{\alpha}) \in T_{1}$ iff $\exists x \in \omega^{\omega} \exists f \in\left(\omega_{1}\right)^{\omega}$ extending $s, \vec{\alpha}$ with $x \in \mathrm{WO}$ and $f$ mapping $\omega$ order preserving (with respect to the ordering given by $x$ ) into $\omega_{1}$. So, WO $=p\left[T_{1}\right] \equiv$ the projection of the set paths through $T_{1}$. Also, if $\alpha<\omega_{1}$ is a limit ordinal and $x \in$ WO with $|x|=\alpha$, then $\left(T_{1}\right)_{x} \mid \alpha$ is ill-founded. Here, $|x|$ is the rank of the well-ordering coded by $x,\left(T_{1}\right)_{x}$ is the "section" of the tree $T_{1}$ at $x$ (i.e. $\left\{\vec{\alpha} \in\left(\omega_{1}\right)^{<\omega}:(x \mid\right.$ length $\left.\left.(\vec{\alpha}), \vec{\alpha}) \in T_{1}\right\}\right)$, and $\left(T_{1}\right)_{x} \mid \alpha$ denoted the restriction of $\left(T_{1}\right)_{x}$ to ordinals less than $\alpha$. Let $T_{2} \subseteq(\omega \times \omega)^{<\omega}$ be a tree with $\neg \mathrm{WO}=p\left[T_{2}\right]$, as $\neg \mathrm{WO}$ is $\Sigma_{1}^{1}$. We then define the "Kunen tree" $\widetilde{T} \subseteq\left(\omega \times \omega_{1} \times \omega \times \omega \times \omega\right)^{<\omega}$ by: $(s, \vec{\alpha}, t, u, v) \in \widetilde{T}$ iff $\left[s, t, u, v \in \omega^{n}\right.$ and $\vec{\alpha} \in \omega_{1}^{n}$ for some $n$, and $\exists x \in \omega^{\omega} \exists \sigma \in \omega^{\omega} \exists y \in \omega^{\omega} \exists z \in \omega^{\omega}$ such that $x, \sigma$, $y, z$ extend $s, t, u, v$ and $\sigma(x)=y,(s, \vec{\alpha}) \in T_{1}$, and $\left.(u, v) \in T_{2}\right]$. Here we are viewing each $\sigma \in \omega^{\omega}$ as coding a strategy, and $\sigma(x)$ is the result of 
following that strategy against play $x$. By coding elements of $\left(\omega_{1} \times \omega \times \omega\right)^{<\omega}$ by elements of $\omega_{1}^{<\omega}$, we may view $\widetilde{T}$ as a tree $T$ on $\omega \times \omega_{1}$. A simple game argument using $\mathrm{AD}$, due to Kunen shows that for any $f: \omega_{1} \rightarrow \omega_{1}$ there is a $\sigma \in \omega^{\omega}$ such that $T_{\sigma}$ is well-founded and such that for almost all $\alpha<\omega_{1}$ with respect to $N, f(\alpha)<\left|T_{\sigma}\right| \alpha \mid$ (play the Solovay game where I plays, $x$, II plays $y$, and II wins iff $x \in \mathrm{WO} \Rightarrow\left(T_{2}\right)_{y}$ is well-founded and $\left|\left(T_{2}\right)_{y}\right|>f(|x|)$, where $|x|<\omega_{1}$ is the ordinal coded by $x$, and $\left|\left(T_{2}\right)_{y}\right|$ denotes the rank of $\left(T_{2}\right)_{y}$. A winning strategy $\sigma$ for II will suffice).

We fix now $\sigma_{0} \in \omega^{\omega}$ such that $T_{\sigma_{0}}$ is well-founded that $\left|T_{\sigma_{0}}\right| \alpha \mid>g_{0}(\alpha)$ for almost all $\alpha$ with respect to $N$, say on the c.u.b. set $D_{0} \subseteq C_{0}$. We let $B_{0} \subseteq A_{0}$ be the measure one (with respect to $\nu$ ) subset of $A_{0}$ consisting of those $\beta \in \omega_{1}$ such that $\alpha<\beta<\left|T_{\sigma_{0}}\right| \alpha \mid$ for some $\alpha \in D_{0}$. By thinning out $D_{0}$, if necessary, we may assume that this $\alpha=\alpha(\beta)$ is uniquely defined and $\beta \in B_{0}$ (i.e. select $D_{0}$ to be closed under the function $F(\alpha)=\left|T_{\sigma_{0}}\right| \alpha \mid$ ).

We define, now, for $\beta \in B_{0}, h(\beta)=$ that $\theta<\alpha(\beta)$ such that $\beta=$ $\left|T_{\sigma_{0}}\right|(\alpha(\beta))(\theta) \mid=$ the rank of $\theta$ in the tree $T_{\sigma_{0}} \mid \alpha(\beta)$. It is immediate that for $\beta_{1} \neq \beta_{2}$ and $\alpha\left(\beta_{1}\right)=\alpha\left(\beta_{2}\right)$, that $h\left(\beta_{1}\right) \neq h\left(\beta_{2}\right)$.

We set $\nu_{1}=h_{0}\left(\nu_{0}\right)$.

If $\nu_{1}$ is not principal, we now repeat the above arguments starting with $\nu_{1}$ instead of $\nu_{0}$. Repeating the argument, we get $\nu_{0}, \nu_{1}, \nu_{2}, \ldots$, functions $h_{0}, h_{1}, h_{2}, \ldots$, etc. with $h_{i}\left(\nu_{i}\right)=\nu_{i+1}$ and $h_{i}(\alpha)<\alpha$ almost everywhere with respect to $\nu_{i}$. Hence, $\left[h_{0}\right]_{\nu_{0}}>\left[h_{1} \circ h_{0}\right]_{\nu_{0}}>\left[h_{2} \circ h_{1} \circ h_{0}\right]_{\nu_{0}}>\cdots$ etc. As we are getting a descending sequence of ordinals, we must reach a principal measure after a finite number of steps. We let $n$ be minimal such that $\nu_{n+1}$ is principal (say on the ordinal $\gamma<\omega_{1}$ ). Thus, we have produced $f_{0}, \ldots, f_{n}$, $g_{0}, \ldots, g_{n}, h_{0}, \ldots, h_{n}, \sigma_{0}, \ldots, \sigma_{n}$, c.u.b. sets $D_{0}, \ldots, D_{n}$ and $B_{0}, \ldots, B_{n}$ with $\nu_{i}\left(B_{i}\right)=1$. In particular $h_{i}\left(\nu_{i}\right)=v_{i+1}$. We let $D=\bigcap_{i=1}^{n} D_{i}$ and $B=$ $B_{0} \cap h_{0}^{-1}\left(B_{1}\right) \cap\left(h_{1} \circ h_{0}\right)^{-1}\left(B_{2}\right) \cap \cdots \cap\left(h_{n} \circ \cdots \circ h_{0}\right)^{-1}\left(B_{n}\right)$. So, $D$ is c.u.b. in $\omega_{1}$, and $\nu_{0}(B)=1$.

We let $N^{r}$ denote the $r$-fold product of the measure $N$.

Claim. For all $A \subseteq \omega_{1}$ we have $\nu(A)=1$ iff for almost all $\left(\alpha_{0}, \ldots, \alpha_{n}\right)$ with respect to $N^{n}$,

$$
\begin{aligned}
& F\left(\sigma_{0}, \ldots, \sigma_{n}, \alpha_{0}, \ldots, \alpha_{n}, \gamma\right) \\
& \quad \equiv\left|T_{\sigma_{0}}\right| \alpha_{n}\left(\left|T_{\sigma_{1}}\right| \alpha_{n-1}\left(\cdots\left(\left|T_{\sigma_{0}}\right| \alpha_{0}(\gamma) \mid\right) \cdots\right) \mid\right) \mid \in A .
\end{aligned}
$$

Proof. We suppose not, and let $A \subseteq \omega_{1}$ have measure one with respect to $\nu$, and $C \subseteq \omega_{1}$ be c.u.b. and such that for all $\alpha_{1}<\alpha_{2}<\cdots<\alpha_{n}$, and $\alpha_{1}, \ldots, \alpha_{n} \in C, F\left(\sigma_{0}, \ldots, \sigma_{n}, \alpha_{0}, \ldots, \alpha_{n}, \gamma\right) \notin A$. Then $\bar{C}=C \cap D$ is also c.u.b. in $\omega_{1}$. It also follows from the definition of the functions $f_{i}$, $g_{i}, h_{i}$ that for almost all $\alpha$ with respect to $\nu$, that $g_{0}(\alpha) \in \bar{C}, g_{1} \circ h_{0}(\alpha) \in$ $\bar{C}, \ldots, g_{n} \circ h_{n-1} \circ h_{n-2} \circ \cdots \circ h_{1}(\alpha) \in \bar{C}$, and $h_{n} \circ h_{n-1} \circ \cdots \circ h_{2} \circ h_{1}(\alpha)=\gamma$. In particular, we must have that for some $\alpha \in A$ and $\alpha_{1}<\alpha_{2}<\cdots<\alpha_{n}$, all 
in $\bar{C}$ that $F\left(\sigma_{0}, \ldots, \sigma_{n}, \alpha_{0}, \ldots, \alpha_{n}, \gamma\right)=\alpha$. This, however, contradicts the definitions of $A, \bar{C}$.

\section{Simple SETS AND A CODING of the SUbSets of $\omega_{1}$}

Ideas of Kunen (see [Solovay], where a coding of the subsets of $\omega_{\omega}$ is given) allow one to convert an analysis of the measures on $\kappa$ into a coding of the subsets of $\kappa$. Doing this in a careful enough manner will allow us to get a coding for the subsets of $\omega_{1}$ sufficient to obtain the strong partition relation on $\omega_{1}$.

To each $\sigma \in \omega^{\omega}$ such that $T_{\sigma}$ (see previous section for the definitions of $T$, $\left.T_{1}, T_{2}\right)$ is well-founded, we associate a c.u.b. $C_{\sigma} \subseteq \omega_{1}$ by : $C_{\sigma}=\left\{\alpha<\omega_{1}: \alpha\right.$ is a limit ordinal and $\left.\forall \beta<\alpha\left(\left|T_{\sigma}\right| \beta \mid<\alpha\right)\right\}$. We claim that for each c.u.b. $C \subseteq \omega_{1}$, there is a $\sigma$ such that $C_{\sigma} \subseteq C$. To see this, play the Solovay game where I plays $x$, II plays $y$, and II wins if $\left(x \in \mathrm{WO} \Rightarrow\left(T_{2}\right)_{y}\right.$ is well-founded and $\left|\left(T_{2}\right)_{y}\right|>$ the next element of $C$ after $|x|=$ the ordinal coded by $\left.x\right)$. By boundedness, II has a winning strategy, say $\sigma . T_{\sigma}$ is well-founded and for $\beta \in \omega_{1},\left|T_{\sigma}\right| \beta \mid>$ the next element of $C$ after $\beta$. Hence, every element of $C_{\sigma}$ is a limit of points in $C$, hence in $C$. We say that a c.u.b. $C \subseteq \omega_{1}$ is codeable if there is a $\sigma \in \omega^{\omega}$ such that $T_{\sigma}$ is well-founded and $C_{\sigma}=C$.

Definition. We say that $S \subseteq \omega_{1}$ is simple if there is a codeable $C \subseteq \omega_{1}$ and reals $\sigma_{1}, \ldots, \sigma_{n}$ with $T_{\sigma_{0}}, \ldots, T_{\sigma_{n}}$ well-founded, and an ordinal $\gamma<\omega_{1}$ such that $S=\left\{\alpha<\omega_{1}: \alpha=F\left(\sigma_{1}, \ldots, \sigma_{n}, \alpha_{0}, \ldots, \alpha_{n}, \gamma\right)\right.$ for some $\alpha_{0}<\cdots<\alpha_{n}$ all in $C$ \} (see previous sections for the notation).

From the claim of the previous section we have that if $\nu$ is a measure and $A \subseteq \omega_{1}$ with $\nu(A)=1$, then $A$ contains a simple set $S$ with $\nu(S)=1$.

We say that $x \in \omega^{\omega}$ codes the simple set $S$ if $x_{0}=\langle n, \sigma\rangle$ for some $n$, $\sigma$ where $T_{\sigma}$ is well-founded and codes $C=C_{\sigma}, T_{x_{1}}, T_{x_{2}}, \ldots, T_{x_{n}}$ are wellfounded, $x_{n+1} \in$ WO with $\left|x_{n+1}\right|=\gamma$ for some $\gamma$, and $S$ is obtained from $C, T_{x_{1}}, \ldots, T_{x_{n}}, \gamma$ as above. Here, via some fixed coding, each real $x$ codes countably many reals $x_{0}, x_{1}, \ldots$, etc.

Theorem $1(A D+D C)$. Every $A \subseteq \omega_{1}$ is a countable union of simple sets.

Proof. We use Kunen's argument. We suppose not, and let $A$ be a counterexample. We let $\mathscr{I}$ be the ideal of sets $I \subseteq \omega_{1}$ such that $I \cap A$ is contained in a countable union of simple sets each of which is contained in $A$, and $I \cap \neg A$ is contained in a countable union of simple sets, each contained in $\neg A$. Clearly $\mathscr{I}$ is a $\sigma$-ideal, and is proper, as $\omega^{\omega} \notin \mathscr{I}$. We let $\mathscr{M}$ denote the Martin measure on the degrees (recall that with AD every set of degrees either contains or omits a cone of degrees; this defines the Martin measure). Using the coding lemma (see [Moschovakis]), we fix a surjection $f: \omega \stackrel{\omega \text { onto }}{\rightarrow} \mathscr{P}\left(\omega_{1}\right)$. We then define $H$ from the degrees $\mathscr{D}$ into $\omega_{1}$ by $H(d)=$ least element of $\omega_{1}$ not in $\bigcup_{x \in d, f(x) \in \mathcal{I}} f(x)$. We let $\nu$ be the measure on $\omega_{1}$ given by $\nu=H(\mathscr{M})$. 
Clearly, then, for any $I \in \mathscr{I}, \nu(I)=0$. Now, either $\nu(A)$ or $\nu(\neg A)=1$. By symmetry, suppose $\nu(A)=1$. Then from $\S 2$, it follows that there is a simple set $S \subseteq A$ with $\nu(S)=1$. This, however, contradicts the fact that $S \in \mathcal{I}$.

If $x \in \omega^{\omega}$ codes a simple set, we let $S_{x}$ denote the simple set it codes. The above theorem gives a coding for the subsets of $\omega_{1}$. Namely, we say $x$ codes $A \subseteq \omega_{1}$ if each $x_{i}$ codes a simple set $S_{x_{i}}$ and $A=\bigcup_{i \in \omega} S_{x_{i}}$. Hence, we have shown that every $A \subseteq \omega_{1}$ gets a code.

Now, in order to get the strong partition on $\omega_{1}$, we require a coding for the functions $f: \omega_{1} \rightarrow \omega_{1}$. Of course, we could view a function as a subset of $\omega_{1} \times \omega_{1}$, and hence via some coding of $\omega_{1} \times \omega_{1}$ into $\omega_{1}$ as a subset of $\omega_{1}$. The coding we get this way, however, does not seem to be quite good enough. We require a slight strengthening of our previous definition.

Definition. We say $g \subseteq \omega_{1} \times \omega_{1}$ is a subfunction if it is a function with domain some $A \subseteq \omega_{1}$ (i.e. $\forall \alpha, \beta, \gamma(\alpha, \beta) \in g$ and $\left.(\alpha, \gamma) \in g \Rightarrow \beta=\gamma\right)$. We say $g$ is a subfunction of $f$ if $g \subseteq f$ as subsets of $\omega_{1} \times \omega_{1}$.

Definition. We say the subfunction $g$ is simple if there is a codeable $C \subseteq \omega_{1}$, $\sigma_{1}, \ldots, \sigma_{n}$ with $T_{\sigma_{1}}, \ldots, T_{\sigma_{n}}$ well-founded and ordinals $\gamma_{1}, \gamma_{2}>\omega_{1}$ such that $g=\left\{\left\langle\alpha_{1}, \alpha_{2}\right\rangle: \exists \beta_{1}<\beta_{2}<\cdots<\beta_{n}, \beta_{1}, \beta_{2}, \ldots, \beta_{n} \in C, \beta_{n} \leq \alpha_{1}\right.$, and $\left.\left\langle F\left(\alpha_{1}, \ldots, \sigma_{n}, \beta_{1}, \ldots, \beta_{n}, \gamma_{1}\right) F\left(\sigma_{1}, \ldots, \sigma_{n}, \beta_{1}, \ldots, \beta_{n}, \gamma_{2}\right)\right\rangle=\left\langle\alpha_{1}, \alpha_{2}\right\rangle\right\}$.

Note " $\beta_{n} \leq \alpha_{1}$ " is the new extra requirement.

In analogy with our previous analysis of subsets of $\omega_{1}$, we now have the following:

Theorem $2(A D+D C)$. Every function $f: \omega_{1} \rightarrow \omega_{1}$ is the countable union of simple subfunctions $f=\bigcup_{i \in \omega} f_{i}$.

Proof. The proof is a slight modification of the proof of Theorem 1. We sketch the differences. We suppose the theorem fails, and fix a counterexample, $f: \omega_{1} \rightarrow \omega_{1}$. We let $\mathscr{I}$ be the ideal on $X \equiv\left\{\left(\alpha_{1}, \alpha_{2}\right): \alpha_{1}, \alpha_{2}<\omega_{1}\right.$ and $\left.f\left(\alpha_{1}\right)=\alpha_{2}\right\}$ consisting of all $I \subseteq X$ such that $I \subseteq \bigcup_{i \in \omega} f_{i}$ where each $f_{i} \subseteq X$ (i.e. $f_{i}$ is a subfunction of $f$ ) and is simple. Clearly $\mathscr{I}$ is a $\sigma$ ideal and is proper, as $X \notin \mathscr{I}$. Arguing as before, we get a measure $\nu$ on $X$ (i.e. $\nu(X)=1$ ) with $\nu(I)=0$ for all $I \in \mathscr{I}$. Let $f_{0}: X \rightarrow \omega_{1}$ be a representative for the least equivalence class which contains a function which is (nonstrictly) pressing down (i.e. $f_{0}\left(\alpha_{1}, \alpha_{2}\right) \leq \alpha_{1}$ for all $\left.\alpha_{1}, \alpha_{2}\right)$, nonconstant almost everywhere, and monotonically increasing with respect to $\alpha_{1}$ (i.e. $\left.\alpha_{1} \leq \bar{\alpha}_{1} \Rightarrow f_{0}\left(\alpha_{1}, \alpha_{2}\right) \leq f_{0}\left(\bar{\alpha}_{1}, \bar{\alpha}_{2}\right)\right)$. It follows as before that $f_{0}(\nu)=N$, the normal measure on $\omega_{1}$. We let

$$
g_{0}(\alpha)=\sup _{\left\{\beta: f_{0}(\beta, f(\beta)) \leq \alpha\right\}} \max (\beta, f(\beta)) .
$$

We choose $\sigma_{0}$ with $T_{\sigma_{0}}$ well-founded such that the function $\alpha \rightarrow\left|T_{\sigma_{0}}\right| \alpha \mid$ dominates $g_{0}$ almost everywhere with respect to $N$. We define the sets $A_{0}$, $B_{0}, C_{0}, D_{0}$ as before $\left(\right.$ so $\left.\nu\left(B_{0}\right)=1\right)$ and $H_{0}: B_{0} \rightarrow \omega_{1}^{2}$ by: $h_{0}\left(\alpha_{1}, \alpha_{2}\right)=$ 
that pair $\left(\bar{\alpha}_{1}, \bar{\alpha}_{2}\right)$ such that $\left|T_{\sigma_{0}}\right| \delta\left(\bar{\alpha}_{1}\right) \mid=\alpha_{1}$ and $\left|T_{\sigma_{0}}\right| \delta\left(\bar{\alpha}_{2}\right) \mid=\alpha_{2}$, where $\delta=f_{0}\left(\alpha_{1}, \alpha_{2}\right)$. As before, we then set $\nu_{1}=h(\nu)$, so $\nu$, is a measure on pairs as well. The remainder of the argument then proceeds as in the proof of Theorem 1; in fact, by coding $\omega_{1}^{2}$ by $\omega_{1}$ in some standard manner (the Gödel ordering for example), we may simply use that analysis here now. At any rate, the procedure eventually terminates with some $\nu_{n+1}$, a principal measure on $\left(\gamma_{1}, \gamma_{2}\right)$, say. Using these $\left(\gamma_{1}, \gamma_{2}\right), \sigma_{0}, \ldots, \sigma_{n}$ and $D \subseteq \omega_{1}$ obtained as in Theorem 1, we now get a simple subfunction $g$ of $f$, with

$$
\nu\left\{\left(\alpha_{1}, \alpha_{2}\right): g\left(\alpha_{1}\right)=\alpha_{2}\right\}=1,
$$

a contradiction. Notice that from the definition of $g_{0}$ here that the simple subfunction we obtain does indeed satisfy the extra requirement " $\beta_{n} \leq \alpha_{1}$ " imposed in the definition of simple. This completes the proof of Theorem 2.

\section{THE STRONG PARTITION RELATION ON $\omega_{1}$}

Theorem 2 allows us to code functions by reals $x \in \omega^{\omega}$ to before. That is, $x$ codes a function, which we denote by $f_{x}$, if each $x_{i}$ codes a simple subfunction $f_{x_{i}}$ and $f=\bigcup_{i \in \omega} f_{x_{i}}$. Here, we code simple subfunctions $f_{w}$ similarly to before. That is $w_{0}=\langle n, \sigma\rangle$ for some $n, \sigma$ with $\sigma$ coding the c.u.b. set $C_{\sigma} \subseteq \omega_{1}, T_{w_{1}}, \ldots, T_{w_{n}}$ are well-founded, $w_{n+1}=\left\langle y_{n+1}, z_{n+1}\right\rangle$ with $y_{n+1}, z_{n+1} \in$ WO so $\left|y_{n+1}\right|=\gamma_{1},\left|z_{n+1}\right|=\gamma_{2}$ for some $\gamma_{1}, \gamma_{2}$, and $C_{\sigma}$, $T_{w_{1}}, \ldots, T_{w_{n}}, \gamma_{1}, \gamma_{2}$ generate $f_{w}$ as in the definition of simple subfunction.

Using this coding, we may now repeat Martin's argument for the strong partition relation on $\omega_{1}$. For the sake of completeness, we give the argument, although we will be somewhat sketchy as Kechris (see [Kechris 2]) gives a detailed presentation of the proof of the partition property assuming the existence of a coding function $f: \omega_{1} \rightarrow \omega_{1}$ satisfying certain properties-which ours does. We require a definition:

Definition. For $x \in \omega^{\omega}$ and $\alpha<\omega_{1}$, we say $x$ codes a function through $\alpha$ iff

(1) $\forall \alpha_{1} \leq \alpha \quad \exists \alpha_{2}<\omega_{1} \quad \exists i \in \omega \quad \exists n \in \omega \quad \exists \beta_{1}<\beta_{2}<\cdots<\beta_{n} \leq \alpha_{1}$ satisfying the following:

(a) $x_{i, 0}=\langle n, \sigma\rangle$ for some $\sigma \in \omega^{\omega}$ (here $x_{i, j}=\left(x_{i}\right)_{j}=$ the $j$ th component of $x_{i}$ ).

(b) $x_{i, n+1}=\langle y, z\rangle$ with $y, z \in \mathrm{WO},|y|=\gamma,\left|z_{0}\right|=\gamma_{2}$ for some $\gamma_{1}, \gamma_{2}<$ $\alpha_{1}$.

(c) for all $1 \leq i \leq n, \forall \delta<\beta_{i}\left(T_{\sigma} \mid \delta\right.$ is well-founded and $\left.\left|T_{\sigma}\right| \delta \mid<\beta_{i}\right)$.

(d) $\left|T_{x_{i, n}}\right| \beta_{1}\left(\gamma_{1}\right)|,| T_{x_{i, n}}\left|\beta_{1}\left(\gamma_{2}\right)\right|$ are well-defined (that is, the subtree of $T_{x_{i, n}} \mid \beta_{1}$ starting at $\gamma_{1}$ is well-founded, and similarly for $\left.\gamma_{2}\right), \ldots,\left|T_{x_{i, 1}}\right|$ $\beta_{n}\left(\left|T_{x_{i, 2}}\right| \beta_{n-1}\left(\cdots\left(\left|T_{x_{i, n}}\right| \beta_{1}\left(\gamma_{1}\right) \mid\right) \cdots\right) \mid\right) \mid$, and the same expression with $\gamma_{2}$ are well-defined.

(e) $F\left(x_{i, 1}, \ldots, x_{i, n}, \beta_{1}, \ldots, \beta_{n}, \gamma_{1}\right)=\alpha_{1}$, $F\left(x_{i, 1}, \ldots, x_{i, n}, \beta_{1}, \ldots, \beta_{n}, \gamma_{2}\right)=\alpha_{2}$ and 
(2) $\forall \alpha_{1} \leq \alpha \quad \forall \alpha_{2}<\omega_{1} \quad \forall i, i^{\prime} \in \omega \quad \forall n, n^{\prime} \in \omega \quad \forall \beta_{1}<\beta_{2}<\cdots<\beta_{n} \leq \alpha_{1}$ $\forall \beta_{1}^{\prime}<\beta_{2}^{\prime}<\cdots<\beta_{n^{\prime}}^{\prime} \leq \alpha_{1}$ if $\left(\alpha_{1}, \alpha_{2}, i, n, \beta_{1}, \ldots, \beta_{n}\right)$ satisfies (a)-(e) in (1) above and $\left(\alpha_{1}, i^{\prime}, n^{\prime}, \beta_{1}^{\prime}, \ldots, \beta_{n^{\prime}}^{\prime}\right)$ satisfies $(\mathrm{a})-(\mathrm{c})$ and

$$
F\left(x_{i^{\prime}, 1}, \ldots, x_{i^{\prime}, n^{\prime}}, \beta_{1}^{\prime}, \ldots, \beta_{n^{\prime}}^{\prime}, \gamma_{i^{\prime}, 1}\right)=\alpha_{1},
$$

then $F\left(x_{i^{\prime}, 1}, \ldots, x_{i^{\prime}, n^{\prime}}, \beta_{1}^{\prime}, \ldots, \beta_{n^{\prime}}^{\prime}, \gamma_{i^{\prime}, 2}\right)$ is defined and equal to $\alpha_{2}$. Here $\gamma_{i^{\prime}, 1}, \gamma_{i^{\prime}, 2}$ are the pair of ordinals coded by $x_{i^{\prime}, n^{\prime}+1}$.

Thus, (1) simply says that $f_{x}(\alpha)$ is defined for all $\alpha \leq \alpha_{1}$ and (2) asserts that this value is unique in a strong sense.

We say that $x$ codes a function through $\alpha$ with values $<\beta$ if $x$ satisfies (1) and (2) above, where in (1) we restrict the $\alpha_{2}$ to be $<\beta$.

Claim. For each $\alpha, \beta<\omega_{1}$, the set $A_{\alpha, \beta} \equiv\left\{x \in \omega^{\omega}: x\right.$ codes a function through $\alpha$ with values $<\beta\}$ is $\Delta_{1}^{1}$.

Proof. We have $x \in A_{\alpha, \beta} \Leftrightarrow \forall \alpha_{1} \leq \alpha \quad \exists \alpha_{2}<\beta[(1): \exists i \in \omega \quad \exists n \in \omega$ $\exists \beta_{1}<\beta_{2}<\cdots<\beta_{n} \leq \alpha_{1}$ such that $\left(\alpha_{1}, \alpha_{2}, i, n, \beta_{1}, \ldots, \beta_{n}\right)$ satisfy (a)-(e) in (1) above, and (2): $\forall i^{\prime} \in \omega \quad \forall n^{\prime} \in \omega \forall \beta_{1}^{\prime}<\beta_{2}^{\prime}<\cdots<$ $\beta_{n^{\prime}}^{\prime}<\alpha_{1}$ if $\left(\alpha_{1}, i^{\prime}, n^{\prime}, \beta_{1}^{\prime}, \ldots, \beta_{n^{\prime}}^{\prime}\right)$ satisfies (a)-(c) in (1) above and $F\left(x_{i^{\prime}, 1}, \ldots, x_{x^{\prime}, n^{\prime}}, \ldots, \beta_{1}^{\prime}, \ldots, \beta_{n^{\prime}}^{\prime}, \gamma_{i^{\prime}, 1}\right)$ is defined and equal to $\alpha_{1}$ then $F\left(x_{i^{\prime}, 1}, \ldots, x_{i^{\prime}, n^{\prime}}, \beta_{1}^{\prime}, \ldots, \beta_{n^{\prime}}^{\prime}, \gamma_{i^{\prime}, 2}\right)$ is defined and equal to $\left.\alpha_{2}\right]$.

Using the closure of $\Delta_{1}^{1}$ under countable unions and intersections, the fact that $\{w: w \in \mathrm{WO}$ and $|w| \leq \alpha\}$ is $\Delta_{1}^{1}$ for any $\alpha<\omega_{1}$, and the fact that $R_{\alpha, \beta, \gamma} \equiv\left\{z: T_{z} \mid \alpha(\beta)\right.$ is well-founded of rank $\left.\gamma\right\}$ is $\Delta_{1}^{1}$ for all $\alpha, \beta, \gamma<\omega_{1}$ (see [Kechris 2] for more details on the tree computations), we easily get that $A_{\alpha, \beta} \in \Delta_{1}^{1}$.

From this point the argument, due to Martin, for getting the strong partition relation is fairly standard. We suppose $A \subseteq\left\{f: f\right.$ is a function from $\omega_{1}$ to $\omega_{1}$ of the correct type $\}$ is a given partition. We consider the game

$$
\begin{array}{rr}
\text { I } & x \\
\text { II } & y
\end{array}
$$

Here I plays $x \in \omega^{\omega}$ and II plays $y \in \omega^{\omega}$. We let $\alpha(x)$ be the least ordinal $<\omega_{1}$ (if one exists) such that for no $\beta<\omega_{1}$ does $x$ code a function through $\alpha(x)$ with values $<\beta$. We similarly define $\alpha(y)$. If both $\alpha(x), \alpha(y)$ are defined, II wins provided $\alpha(y) \geq \alpha(x)$. If only one is defined, that player loses. If neither is defined, then $x, y$ code functions $f_{x}, f_{y}: \omega_{1} \rightarrow \omega_{1}$. We define $f: \omega_{1} \rightarrow \omega_{1}$ by

$$
f(\alpha)=\sup _{\beta<\omega \cdot(\alpha+1)} \max \left\{f_{x}(\beta), g_{x}(\beta)\right\} .
$$

Then II wins provided $f \in A$.

We suppose that II wins, say by $\sigma$, and find a c.u.b. $C \subseteq \omega_{1}$ homogeneous for the partition - the case of I winning being similar. We let, as above, for $\alpha$, $\beta<\omega_{1}, A_{\alpha, \beta}=\left\{x \in \omega^{\omega}: x\right.$ codes a function through $\alpha$ with values $\left.<\beta\right\}$. 
In particular, for $x \in A_{\alpha, \beta}, \alpha(x)>\alpha$. Hence, $\alpha(\sigma(x))>\alpha$ for all $x \in A_{\alpha, \beta}$. Hence $f_{\sigma(x)}(\alpha)$ is defined for all $x \in A_{\alpha . \beta}$. We claim that

$$
g(\alpha, \beta) \equiv\left(\sup _{x \in A_{\alpha, \beta}} f_{\sigma(x)}(\alpha)\right)<\omega_{1} .
$$

If this were to fail, it is easy to see that we would get a $\Sigma_{1}^{1}$ well-founded relation of length $\omega_{1}$, a contradiction. To be specific, we consider the relation $z \prec w \Leftrightarrow z, w \in \sigma^{\prime \prime} A_{\alpha, \beta}$ and $f_{z}(\alpha)<f_{w}(\alpha) \Leftrightarrow \exists x_{1}, x_{2} \quad\left(x_{1}, x_{2} \in A_{\alpha, \beta}\right.$ and $\left.\sigma\left(x_{1}\right)=z, \sigma\left(x_{2}\right)=w\right)$ and $\exists i, n \in \omega \quad \exists \beta_{1}<\beta_{2}<\cdots<\beta_{n} \leq \alpha$ $\exists j, m \in \omega \quad \exists \gamma_{1} \leq \gamma_{2}<\cdots<\gamma_{m} \leq \alpha \quad\left(F\left(z_{i, 1}, \ldots, z_{i, n}, \beta_{1}, \ldots, \beta_{n}, z_{n+1,1}\right)\right.$ and $F\left(w_{j, 1}, \ldots, w_{j, m}, \gamma_{1}, \ldots, \gamma_{m}, w_{m+1,2}\right)$ are defined and equal to $\alpha$ and “ $F\left(z_{i, 1}, \ldots, z_{i, n}, \beta_{1}, \ldots, \beta_{n}, z_{n+1,2}\right)<F\left(w_{j, 1}, \ldots, w_{j, m}, \gamma_{1}, \ldots, \gamma_{m}\right.$, $\left.w_{m+1,2}\right)$ "). Everything except for the last inequality in quotation marks is easily seen to be $\Sigma_{1}^{1}$. For this, we use a $\Sigma_{1}^{1}$ relation which when restricted to $\{(z, w)$ : $F\left(z_{i, 1}, \ldots, z_{n+1,2}\right), F\left(w_{j, 1}, \ldots, w_{m+1,2}\right)$ are defined $\}$ correctly computes the inequality in quotation marks. This again follows easily from the Sierpinski tree computations (see again [Kechris 2] for details).

We let, now $D \subseteq \omega_{1}$ be a c.u.b. set closed under $g$, i.e. if $\gamma \in D$ and $\alpha, \beta<\gamma$ then $g(\alpha, \beta)<\gamma$. Finally, we let $C \subseteq \omega_{1}$ be the c.u.b. set consisting of the limit points of $D$. We claim that if $f: \omega_{1} \rightarrow C$ is of the correct type, then $f \in A$. For any such $f$, we may find an $x \in \omega^{\omega}$ coding a function $f_{x}$ (so $\alpha(x)$ is not defined) such that range $f_{x} \subseteq D$ and $f(\alpha)=$ $\sup _{\beta<\omega \cdot(\alpha+1)} f_{x}(\beta)$. We play this for I against II's winning strategy $\sigma$, producing $y=\sigma(x)$. So, $f_{y}(\alpha)$ is defined for all $\alpha<\omega_{1}$, and from the definition of $D$ it follows that $\sup _{\beta<\omega \cdot(\alpha+1)} f_{y}(\beta) \leq f(\alpha)$ for all $\alpha<\omega_{1}$, and from the definition of the partition it now follows that $f \in A$. This completes the proof of the strong partition relation on $\omega_{1}$.

\section{REFERENCES}

S. Jackson, A calculation of $\boldsymbol{\delta}_{5}^{1}$, Ph.D. thesis, UCLA, 1983.

_. $A D$ and the projective ordinals, Cabal Seminar 81-85, Lecture Notes in Math., vol. 1333, Springer-Verlag, Berlin, 1988, pp. 117-220.

A. S. Kechris, $A D$ and infinite exponent partition relations, unpublished manuscript, Dec. 1977. , AD and projective ordinals, Cabal Seminar 76-77, Lecture Notes in Math., vol. 689, SpringerVerlag, Berlin, 1978, pp. 91-132.

A. S. Kechris and H. Woodin, Generic codes for uncountable ordinals, partition properties, and elementary embeddings, (unpublished manuscript).

D. A. Martin, Unpublished notes on the strong partition relation on $\omega_{1}$.

Y. N. Moschovakis, Descriptive set theory, North-Holland, Amsterdam, 1980.

R. M. Solovay, $A \Delta_{3}^{1}$ coding of the subsets of $\omega^{\omega}$, Cabal Seminar 76-77, Lecture Notes in Math., vol. 689, Springer-Verlag, Berlin, 1978, pp. 133-150.

Department of Mathematics, 253-37, California Institute of Technology, Pasadena, CALIFORNIA 91125

Department of Mathematics, University of North Texas, Denton, Texas 76203 